\title{
Morita equivalence of semirings with local units
}

\author{
M. Das, S. Gupta, and S. K. Sardar
}

Communicated by A. V. Zhuchok

\begin{abstract}
In this paper we study some necessary and sufficient conditions for two semirings with local units to be Morita equivalent. Then we obtain some properties which remain invariant under Morita equivalence.
\end{abstract}

\section{Introduction}

The classical Morita theory for rings has been recognized as one of the most important and fundamental tools in studying the structure of rings. In 1958 Morita [13] established the Morita equivalence theory for rings with identity. In 1983, Abrams [1] made a first step in extending the theory of Morita equivalence to rings without identity. He considered rings with a commuting set of idempotents such that every element of the ring admits one of these idempotents as a two-sided identity and studied the equivalence of the categories of all unitary left modules of these rings. Ánh and Márki [4] further generalized Abrams' result to rings with local units by weakening the condition of commutativity of idempotents. In the year 2011, Katsov and Nam [10] transferred the ring theoretic approach of Morita equivalence to semirings with identity. In [15], Sardar et al. connected Morita equivalence of semirings with a new and equivalent version of Morita context for semirings. Later Katsov et al. [11], Sardar and Gupta $[16,17]$ independently studied some properties of semirings

The first author is grateful to CSIR, Govt. of India, for providing research support. 2020 MSC: 16Y60, 16 Y99.

Key words and phrases: Morita equivalence, Morita context, Morita invariant, semiring, semimodule. 
which remain invariant under Morita equivalence. The aim of this paper is to extend the theory to cover a wider range of semirings namely the semirings with local units in the sense that any two elements of the semiring have a common two sided identity. In order to develop this theory we consider the category $R$-Sem consisting of all unitary left $R$-semimodules $M$ i.e., semimodules ${ }_{R} M$ such that $R M=M$, where $R$ is a semiring with local units and say two such semirings $R$ and $S$ to be Morita equivalent if the categories $R$-Sem and $S$-Sem are equivalent. Since for a semiring $A$ with identity, $A$-Sem coincides with the category $A$-SEM of all left $A$-semimodules, our notion of Morita equivalence coincides with that of semiring with identity [10]. Consequently, some of the results of Katsov et al. [10] are encompassed in their counterparts obtained here. We organize the paper as follows. In Section 2 we recall some necessary preliminaries on semirings and semimodules. In Section 3 we define locally projective unitary $R$-semimodule and present some characterizing properties of locally projective generators in semimodule categories. In Section 4 we develop some tools to investigate some necessary and sufficient conditions for $R$-Sem and $S$-Sem to be equivalent. Analogously to the case of semirings with identity we show that two semirings with local units $R$ and $S$ are Morita equivalent if and only if there exists a unitary Morita context $(R, S, P, Q, \tau, \mu)$ with $\tau, \mu$ surjective. We also identify the semirings with local units which are Morita equivalent to semirings with identity (cf. Prop. 4.14). Finally we conclude the paper by studying some properties of semirings preserved under Morita equivalence in Section 5.

\section{Preliminaries}

A semiring ${ }^{1}[6]$ is a nonempty set $R$ on which operations of addition and multiplication have been defined such that $(1)(R,+)$ is a commutative monoid with identity element $0,(2)(R, \cdot)$ is a semigroup, (3) multiplication distributes over addition from either side, (4) $0 r=0=r 0$ for all $r \in R$. A left $R$-semimodule over a semiring $R$ is a commutative monoid $\left(M,+, 0_{M}\right)$ together with a scalar multiplication from $R \times M$ to $M$, denoted by $(r, m) \mapsto r m$, which satisfies the following identities: (1) $\left(r+r^{\prime}\right) m=$ $r m+r^{\prime} m,(2) r\left(m+m^{\prime}\right)=r m+r m^{\prime}$, (3) $\left(r r^{\prime}\right) m=r\left(r^{\prime} m\right)$, (4) $r 0_{M}=$ $0_{M}=0 m$ for all $r, r^{\prime} \in R$ and $m, m^{\prime} \in M$. Right $R$-semimodules and $R$-S-bisemimodules are defined analogously. We will distinguish left and right $R$-semimodules by writing ${ }_{R} M$ and $M_{R}$, respectively. Let $M$ and $N$

\footnotetext{
${ }^{1}$ Although Golan called it a hemiring, we call it semiring in the present article.
} 
be two left $R$-semimodules. Then a monoid homomorphism $f: M \rightarrow N$ is called a left $R$-homomorphism if $f(r m)=r f(m)$ for all $r \in R$ and $m \in M$. The set of all $R$-morphisms from $M$ to $N$ is denoted by $\operatorname{Hom}_{R}(M, N)$, in particular $\operatorname{End}_{R}(M)$ denotes the set of all $R$-morphisms from $M$ to itself. Right $R$-homomorphisms and bisemimodule homomorphisms are defined analogously.

A semiring $R$ (semimodule $P$ ) is called additively cancellative [7] if $a+x=a+y$ implies $x=y$ for all $a, x, y \in R$ (respectively $a, x, y \in P$ ) and called additively idempotent [7] if $a+a=a$ for all $a \in R$ (respectively $a \in P$ ). If for each element $a$ of a semiring $R$ (semimodule $P$ ) there exists an element $b \in R$ (respectively $b \in P$ ) such that $a+b+a=a$ the semiring (respectively semimodule) is said to be additively regular [6]. A semiring $R$ (semimodule $P$ ) is said to be zero-sum free [7] if $a+b=0$ implies $a=b=0$ for all $a, b \in R$ (respectively $a, b \in P$ ). A nonempty subset $I$ of a semiring $R$ is called an ideal [6] of $R$ if $i+j \in I$ and $r i, i r \in I$ for any $i, j \in I$ and $r \in R$. A semiring (semimodule) is said to be Noetherian [6] if any ascending chain of ideals (respectively subsemimodules) terminates.

Now we recall some preliminaries related to $k$-ideals and $h$-ideals. An ideal $I$ of a semiring $R$ is called a $k$-ideal [8] (also called subtractive ideal in [6]) of $R$ if for $x \in I, y \in R, x+y \in I$ implies $y \in I$. A subsemimodule $N$ of a semimodule $P$ is called a $k$-subsemimodule ${ }^{1}$ (called subtractive subsemimodule in [6]) of $P$ if for $x \in N, y \in P, x+y \in N$ implies $y \in N$. An ideal $I$ of a semiring $R$ is called an $h$-ideal [8] of $R$ if for $y_{1}, y_{2} \in I, x, z \in R, x+y_{1}+z=y_{2}+z$ implies $x \in I$. A subsemimodule $N$ of a semimodule $P$ is called an $h$-subsemimodule [14] of $P$ if for $y_{1}, y_{2} \in N, x, z \in P, x+y_{1}+z=y_{2}+z$ implies $x \in N$. The $k$ closure [8] of an ideal $I$ (a subsemimodule $N$ ) is denoted by $\bar{I}$ (respectively $\bar{N}$ ) and is defined by $\bar{I}=\{x \in R \mid x+i \in I$ for some $i \in I\}$ (respectively $\bar{N}=\{x \in P \mid x+p \in N$ for some $p \in N\}$ ). The $h$-closure [8] of an ideal $I$ (a subsemimodule $N$ ) is denoted by $\tilde{I}$ (respectively $\tilde{N}$ ) and is defined by $\tilde{I}=\left\{x \in R \mid x+y_{1}+z=y_{2}+z\right.$ for some $\left.y_{1}, y_{2} \in I, z \in R\right\}$ (respectively $\tilde{N}=\left\{x \in P \mid x+p_{1}+z=p_{2}+z\right.$ for some $\left.\left.p_{1}, p_{2} \in N, z \in P\right\}\right)$.

An ideal $I$ of a semiring $R$ defines a congruence $\mathcal{B}_{I}$ on $R$, called the Bourne congruence [6], given by $r \mathcal{B}_{I} r^{\prime}$ if and only if there exist $a, a^{\prime} \in I$ satisfying $r+a=r^{\prime}+a^{\prime}$. Similarly $I$ defines another congruence $\mathcal{I}_{I}$ on $R$, called the Iizuka congruence [6], given by $r \mathcal{I}_{I} r^{\prime}$ if and only if there exist $a, a^{\prime} \in I$ and $s \in R$ satisfying $r+a+s=r^{\prime}+a^{\prime}+s$. A congruence $\rho$ on a semiring $R$ is called a ring congruence [5] if the factor semiring $R / \rho$ is a

\footnotetext{
${ }^{1}$ In the present article subtractiveness is replaced by $k$.
} 
ring. A subsemimodule $N$ of a semimodule $P$ defines a congruence $\mathcal{B}_{N}$ on $P$, called the Bourne congruence [6], given by $p \mathcal{B}_{N} p^{\prime}$ if and only if there exist $a, a^{\prime} \in N$ satisfying $p+a=p^{\prime}+a^{\prime}$. Similarly $N$ defines another congruence $\mathcal{I}_{N}$ on $P$, called the Iizuka congruence [6], given by $p \mathcal{I}_{N} p^{\prime}$ if and only if there exist $a, a^{\prime} \in N$ and $p^{\prime \prime} \in P$ satisfying $p+a+p^{\prime \prime}=p^{\prime}+a^{\prime}+p^{\prime \prime}$.

For preliminaries on category theory we refer to [2], [9] and [12].

We adopt the following notions from Ánh and Márki [4].

Definition 2.1. Let $R$ be a semiring and $E(R)$ be a set of idempotents of $R$. Then $R$ is said to be a semiring with local units if every finite subset of $R$ is contained in a subsemiring of the form $e R e$ where $e \in E(R)$ or equivalently if for any finite number of elements $r_{1}, r_{2}, \ldots, r_{n} \in R$, there exists $e \in E(R)$ such that $e r_{i}=r_{i}=r_{i} e$ for all $i=1,2, \ldots, n$. In this case $E(R)$ is a set of local units (slu) of $R$.

Here we give some examples of semirings with local units.

Example 2.2. 1. Suppose $L$ is a distributive lattice with the least element 0 but with no greatest element ${ }^{1}$. Consider $L$ together with the addition + and multiplication $\cdot$ defined by $a+b=\sup \{a, b\}$ and $a \cdot b=\inf \{a, b\}$ respectively, for $a, b \in L$. Then $(L,+, \cdot)$ is a semiring with additive identity 0 but with no multiplicative identity. But it is a semiring with local units, as for any two elements $a, b \in L$, by the absorption law, $a \cdot(a+b)=a=$ $(a+b) \cdot a$ and $b \cdot(a+b)=b=(a+b) \cdot b$, i.e., $a+b$ acts as the common two-sided identity of $a$ and $b$.

2. Let $S$ be a semiring with identity, $X$ be an infinite set and $R=$ $\{f \mid f: X \rightarrow S$ has finite support $\}$. Then $R$ together with the operations $(f+g)(x):=f(x)+g(x)$ and $(f g)(x):=f(x) g(x)$ for $f, g \in R$ and $x \in X$ is a semiring without multiplicative identity. But it is a semiring with local units in view of the following reasons. Suppose $f, g \in R$ with finite supports $\operatorname{supp}(f)$ and $\operatorname{supp}(g)$ respectively, define $h: X \rightarrow S$ by $h(x)=1$ if $x \in \operatorname{supp}(f) \cup \operatorname{supp}(g)$ and $h(x)=0$ otherwise, then for $x \in \operatorname{supp}(f), f h(x)=f(x) h(x)=f(x)$ and for $x \in X \backslash \operatorname{supp}(f), f h(x)=$ $f(x) h(x)=0 \cdot h(x)=0=f(x)$. By a similar argument $h f=f$ and hence $f h=f=h f$ and similarly $g h=g=h g$, i.e., $h$ acts as a two-sided identity of $f$ and $g$.

Definition 2.3. A left $R$-semimodule $M$ over $R$ is said to be unitary if $R M=M$ i.e., for each $m \in M$, there exist $r_{1}, r_{2}, \ldots, r_{n} \in R$ and $m_{1}, m_{2}$, $\ldots, m_{n} \in M$ such that $m=r_{1} m_{1}+r_{2} m_{2}+\cdots+r_{n} m_{n}$.

\footnotetext{
${ }^{1}(\mathbb{N}, l c m, g c d),(\mathbb{N}, \max , \min )$, where $\mathbb{N}$ is the set of all non-negative integers, are some examples of such lattices.
} 
Remark 2.4. If $R$ is a semiring with slu $E$ and $M$ is a unitary $R$ semimodule then for each $m \in M, m=r_{1} m_{1}+r_{2} m_{2}+\cdots+r_{n} m_{n}$ for some $r_{1}, r_{2}, \ldots, r_{n} \in R, m_{1}, m_{2}, \ldots, m_{n} \in M$. Now for $r_{1}, r_{2}, \ldots, r_{n} \in R$, there exists $e \in E$ such that $e r_{i}=r_{i}$ for all $i=1,2, \ldots, n$, therefore $m=\sum_{i=1}^{n} r_{i} m_{i}=\sum_{i=1}^{n} e r_{i} m_{i}=e m$. Thus for every finite subset $M^{\prime} \subset M$ there exists an $e \in E$ such that $e M^{\prime}=M^{\prime}$.

By $R$-Sem we denote the category of unitary left $R$-semimodules together with usual $R$-morphisms.

\section{Locally projective generators}

In what follows unless otherwise mentioned any semiring is with local units and homomorphisms of semimodules are written opposite the scalars.

Definition 3.1. [6] Let $R$ be a semiring with local units. A semimodule $P \in R$-Sem is said to be projective if for a surjective $R$-morphism $\phi: M \rightarrow$ $N$ and an $R$-morphism $\alpha: P \rightarrow N$ in $R$-Sem there exists an $R$-morphism $\bar{\alpha}: P \rightarrow M$ satisfying $\bar{\alpha} \phi=\alpha$.

Recall that [10], for any $R$-semimomodule $P$, the trace ideal $\operatorname{tr}(P):=$ $\sum_{q \in \operatorname{Hom}_{R}(P, R)} P q \subseteq R$.

Proposition 3.2. Let $R$ be a semiring with local units. For any semimodule $P \in R$-Sem, the following are equivalent:

(1) $\operatorname{tr}(P)=R$.

(2) There exists a surjective $R$-morphism $\phi: \bigoplus_{I} P \rightarrow R$ for some index set $I$.

(3) For every semimodule $M \in R$-Sem, there exists a surjective $R$ morphism $\psi: \bigoplus_{\Lambda} P \rightarrow M$ for some index set $\Lambda$.

Proof. (1) $\Rightarrow(2)$ Consider the family of all $R$-morphisms, $f_{\alpha}: P \rightarrow$ $R$. Now if we set $I=\operatorname{Hom}_{R}(P, R)$, then the coproduct induced map $f=\bigoplus_{I} f_{\alpha}: \bigoplus_{I} P \rightarrow R$ is a surjective $R$-morphism since $\left(\bigoplus_{I} P\right) f=$ $\sum_{f_{\alpha} \in I} P f_{\alpha}=\operatorname{tr}(P)=R$.

$(2) \Rightarrow(3)$ Suppose there exists a surjective $R$-morphism $\phi: \bigoplus_{I} P \rightarrow R$ for some index set $I$. Now let $M \in R$-Sem, then for each $m \in M$ consider the map $\rho_{m}: R \rightarrow M$ defined by $r \mapsto r m$, then the coproduct induced map $\rho=\bigoplus_{m \in M} \rho_{m}: \bigoplus_{M} R \rightarrow M$ is a surjective $R$-morphism since $\left(\bigoplus_{M} R\right) \rho=\sum_{m \in M} R \rho_{m}=\sum_{m \in M} R m=M$. Then the direct sum $\phi^{\prime}=\bigoplus_{M} \phi: \bigoplus_{M}\left(\bigoplus_{I} P\right) \rightarrow \bigoplus_{M} R$ is a surjection. Hence $\psi=\phi^{\prime} \rho:$ $\bigoplus_{\Lambda} P \rightarrow M$ is a surjective $R$-morphism where $\Lambda=\dot{\bigcup}_{M} I^{1}$.

${ }^{1} \cup \dot{U}$ denotes the disjoint union 
(2) follows trivially from (3).

$(2) \Rightarrow(1)$ Suppose there exists a surjective $R$-morphism $\phi: \bigoplus_{I} P \rightarrow R$ for some index set $I$. Consider the natural inclusions $\iota_{i}: P \rightarrow \bigoplus_{I} P$ for all $i \in I$. Now for each $i \in I$, let $\phi_{i}=\iota_{i} \phi$, then $R=\left(\bigoplus_{I} P\right) \phi=\sum_{i \in I} P \phi_{i} \subseteq$ $\sum_{q \in \operatorname{Hom}_{R}(P, R)} P q=\operatorname{tr}(P)$. Hence $\operatorname{tr}(P)=R$.

Definition 3.3. A semimodule $P \in R$-Sem is said to be a generator for the category $R$-Sem if $P$ satisfies the equivalent conditions of Prop. 3.2.

Let $R$ be a semiring with local units. Let $M$ be a unitary left $R$ semimodule and $A$ be a subset of $M$. Then $R A=\left\{r_{1} a_{1}+r_{2} a_{2}+\cdots+\right.$ $r_{n} a_{n} \mid n \in \mathbb{N}, r_{i} \in R, a_{i} \in A$, for all $\left.i=1,2, \ldots, n\right\}$ is the subsemimodule generated by $A$. If $A$ generates all of the semimodule $M$ then $A$ is a set of generators for $M$. A unitary $R$-semimodule $M$ is said to be finitely generated if it has a finite set of generators.

We skip the proof of the following proposition as it is analogous to that of its counterpart in module theory (see [3, Proposition 10.1]).

Proposition 3.4. If $M$ is a finitely generated unitary left $R$-semimodule then the following hold:

(1) For every set $\mathcal{A}$ of subsemimodules of $M$ that spans $M$, there is a finite set $\mathcal{F} \subseteq \mathcal{A}$ that spans $M$.

(2) Every semimodule that generates $M$ finitely generates $M$.

Lemma 3.5. Retract of a projective unitary $R$-semimodule is projective.

Proof. Using the definition of projectivity in $R$-Sem (cf. Def. 3.1), the result follows easily from the proof of [9, Prop. 1.7.30] by replacing the notion of epimorphism by surjectivity.

The next result is simply a restatement of Prop 17.19 of [6] in the special case of the category $R$-Sem of $R$-semimodules where $R$ is a semiring with local units.

Proposition 3.6. If $\left\{P_{i} \mid i \in \Omega\right\}$ is a family of unitary left $R$-semimodules then $P=\bigoplus_{i \in \Omega} P_{i}$ is projective if and only if each $P_{i}$ is projective.

Proposition 3.7. ${ }_{R} P$ is a finitely generated projective unitary semimodule if and only if there exists an idempotent $e \in R$ such that $P$ is a retract of $(R e)^{n}, n \geqslant 1$.

Proof. Suppose $P$ is a finitely generated projective unitary semimodule. If $P=\{0\}$ then the zero map $\theta: R e \rightarrow P$ is a retraction in $R$-Sem. So 
we assume that $P \neq\{0\}$ and $\left\{p_{1}, p_{2}, \ldots, p_{n}\right\}$ is a spanning set of ${ }_{R} P$. Then there exists $e^{2}=e \in R$ such that $e p_{i}=p_{i}$ for all $i=1,2, \ldots, n$. Consider $\phi:(R e)^{n} \rightarrow P$ defined by $\left(x_{1}, x_{2}, \ldots, x_{n}\right) \phi=\sum_{i=1}^{n} x_{i} p_{i}$. Since for any $p \in P$ there exist $r_{1}, r_{2}, \ldots, r_{n} \in R$ such that $p=\sum_{i=1}^{n} r_{i} p_{i}$, $\left(r_{1} e, r_{2} e, \ldots, r_{n} e\right) \phi=\sum_{i=1}^{n} r_{i} e p_{i}=\sum_{i=1}^{n} r_{i} p_{i}=p$. Thus $\phi$ is onto. Now $P$ being projective there exists $h: P \rightarrow(R e)^{n}$ such that $h \phi=i d_{P}$. Conversely, suppose $\psi:(R e)^{n} \rightarrow P$ is a retraction in $R$-Sem. Let $f: A \rightarrow$ $B$ be a surjection in $R$-Sem and $g: R e \rightarrow B$ be an $R$-morphism. Define $\bar{g}: R e \rightarrow A$ by $t \mapsto t a$, where $t \in R e$ and $a \in A$ such that $a f=e g$ (if there are more than one $a \in A$ with $a f=e g$ then we choose any one of them and fix it throughout). Then $\bar{g} f=g$, hence $R e$ is projective. Therefore by Prop. 3.6, $(R e)^{n}$ is projective and from Lemma $3.5,{ }_{R} P$ is projective. Also since $(R e)^{n}$ has a finite spanning set $\left\{e_{i}: i=1,2, \ldots, n\right\}$, where each $e_{i}=(0, \ldots, e, \ldots, 0)$, with $e$ in the $i$-th place for all $i=1,2, \ldots, n, P$ is spanned by $\left\{e_{i} \psi: i=1,2, \ldots, n\right\}$. Thus ${ }_{R} P$ is finitely generated.

The notions introduced in the following two definitions are adopted from Ánh and Márki [4].

Definition 3.8. Let $I$ be a partially ordered set such that for each $i, j \in I$ there exists $k \in I$ with $i, j \leqslant k$ and $\left(M_{i}\right)_{i \in I}$ a family of unitary $R$ semimodules. Then $\left(M_{i}\right)_{i \in I}$ is said to be a direct system if for any $i \leqslant j$ we have $R$-morphism $\phi_{i j}: M_{i} \rightarrow M_{j}$ such that $\phi_{i i}=1_{M_{i}}$ for all $i \in I$ and $\phi_{i j} \phi_{j k}=\phi_{i k}$ for $i \leqslant j \leqslant k$.

Moreover a direct system $\left(M_{i}\right)_{i \in I}$ is called a split direct system if for each $i \leqslant j$ in $I$ there exists $\psi_{j i}: M_{j} \rightarrow M_{i}$ such that $\phi_{i j} \psi_{j i}=1_{M_{i}}$ and $\psi_{k j} \psi_{j i}=\psi_{k i}$ for $i \leqslant j \leqslant k$. In this case it follows that $\psi_{i i}=1_{M_{i}}$.

Definition 3.9. A unitary $R$-semimodule $M$ is said to be locally projective if it is the direct limit of a split direct system $\left(M_{i}\right)_{i \in I}$ consisting of subsemimodules that are finitely generated projective.

Proposition 3.10. The $R$-semimodule ${ }_{R} R$ is a locally projective generator.

Proof. Let $E$ be a set of local units of $R$. Define a binary relation $\leqslant$ on $E$ by $e \leqslant f$ if and only if $e f=f e=e$. Then clearly $\leqslant$ is a partial order relation on $E$ and $R$ being a semiring with local units $(E, \leqslant)$ is an upward directed set. Now for each idempotent $e \in R$ and for each pair $e, f \in R$ with $e \leqslant f$ consider the map $\psi_{f e}: R f \rightarrow R e$ given by $r^{\prime} \mapsto r^{\prime} e$, where $r^{\prime} \in R f$ and the natural inclusion maps $\phi_{e}: R e \rightarrow R$ and $\phi_{e f}: R e \rightarrow R f$. Then $(R e)_{e \in E}$ is a split direct system in $R$-Sem and $R=\lim _{E} R e$ where $R e$ is finitely 
generated projective (as seen in the proof of Prop. 3.7) $R$-semimodule for each $e \in E$. Hence $R$ is locally projective. Also for any unitary $R$ semimodule $M$ and for each $m \in M$ consider the map $\rho_{m}: R \rightarrow M$ defined by $r \mapsto r m$, then we have $\rho=\bigoplus_{m \in M} \rho_{m}: \bigoplus_{M} R \rightarrow M$, where $\left(\bigoplus_{M} R\right) \rho=\sum_{m \in M} R \rho_{m}=\sum_{m \in M} R m=M$, which implies that $\rho$ is a surjection. Therefore $R$ is a generator in $R$-Sem.

Proposition 3.11. Let $M$ be a locally projective unitary $R$-semimodule, then every finitely generated subsemimodule $P$ of $M$ is contained in a finitely generated projective subsemimodule of $M$.

Proof. Let $M$ be a locally projective unitary $R$-semimodule. Then there exists a split direct system (cf. Definition 3.8) $\left(M_{i}\right)_{i \in I}$ of finitely generated projective subsemimodules of $M$ such that $M=\lim _{I} M_{i}$. Let $M^{\prime}=$ $\dot{\cup} M_{i} / \rho$, where $\rho$ on $\dot{\cup} M_{i}$ is given by $(x, i) \rho(y, j)$ if and only if there exists $k \in I, i, j \leqslant k$ such that $x \phi_{i k}=y \phi_{j k}$, where $i, j \in I, x \in M_{i}, y \in M_{j}$. Using the existence of $\psi_{j^{\prime} i^{\prime}}$ for each $i^{\prime}, j^{\prime} \in I, i^{\prime} \leqslant j^{\prime}$, it then easily follows that $(x, i) \rho(y, j)$ if and only if $x \phi_{i k}=y \phi_{j k}$ for all $k \in I, i, j \leqslant k$. Now it is a routine matter to verify that $M^{\prime}$ together with the family of $R$ morphisms $\phi_{i}: M_{i} \rightarrow M^{\prime}$ given by $x \mapsto[(x, i)]_{\rho}$ is the direct limit of the split direct system $\left(M_{i}\right)_{i \in I}$. Let $P$ be a subsemimodule of $M$ with a finite spanning set $\left\{p_{1}, p_{2}, \ldots, p_{n}\right\}$. Then identifying $M$ with $M^{\prime}$ we have $p_{k}=\left[\left(x_{k}, i_{k}\right)\right]_{\rho}$ for each $k=1,2, \ldots, n$ where $i_{k} \in I, x_{k} \in M_{i_{k}}$. Let $t \in I$ such that $i_{k} \leqslant t$ for all $k=1,2, \ldots, n$. Then for each $k=1,2, \ldots, n$ we have $p_{k}=x_{k} \phi_{i_{k}}=x_{k} \phi_{i_{k} t} \phi_{t} \in M_{t} \phi_{t}$. Therefore $P \subseteq M_{t} \phi_{t} \cong M_{t}$, where $M_{t}$ is a finitely generated projective subsemimodule of $M$. Hence the proof is complete.

We observe that if $R$ and $S$ are semirings with local units and $U_{S}$ and ${ }_{R} V_{S}$ are unitary then $\operatorname{Hom}_{S}(U, V)$ is a left $R$-semimodule by putting, for $\phi \in \operatorname{Hom}_{S}(U, V)$ and $r \in R,(r \phi)(u)=r \phi(u)$ for $u \in U$. The subsemimodule $R \operatorname{Hom}_{S}(U, V)$ is the largest unitary $R$-subsemimodule of $\operatorname{Hom}_{S}(U, V)$.

Proposition 3.12. Suppose $R$ is a semiring with slu E. Then $\rho: 1_{R \text {-Sem }} \rightarrow R \operatorname{Hom}_{R}\left(R,,_{-}\right)$is a natural isomorphism where for each $M \in R$-Sem, $\rho_{M}: M \rightarrow R \operatorname{Hom}_{R}(R, M)$ is given by $m \mapsto m \rho_{M}(r \mapsto r m)$. For $M^{\prime} \in R$-Sem and $f \in \operatorname{Hom}_{R}\left(M, M^{\prime}\right), \quad \rho_{f}: R \operatorname{Hom}_{R}(R, M) \rightarrow$ $R \operatorname{Hom}_{R}\left(R, M^{\prime}\right)$ is given by $\gamma \mapsto \gamma f$. 
Proof. Clearly $\rho_{M}$ is an $R$-morphism. Also the following diagram commutes:

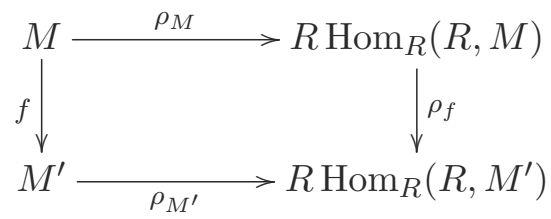

since $r\left(\left(m \rho_{M}\right) \rho_{f}\right)=r\left(m \rho_{M} f\right)=(r m) f=r(m f)=r\left((m f) \rho_{M^{\prime}}\right)$. Hence $\rho$ is a natural transformation. For $M \in R$-Sem, let $m_{1}, m_{2} \in M$, such that $m_{1} \rho_{M}=m_{2} \rho_{M}$. Now since there exists $e \in E$ such that $e m_{1}=$ $m_{1}, e m_{2}=m_{2}$, we have $m_{1}=e\left(m_{1} \rho_{M}\right)=e\left(m_{2} \rho_{M}\right)=m_{2}$. Hence $\rho_{M}$ is injective. Now let $r f \in R \operatorname{Hom}_{R}(R, M)$ and suppose $(r) f=m \in M$ then for any $t \in R, t\left(m \rho_{M}\right)=t m=t((r) f)=(\operatorname{tr}) f=t(r f)$ i.e., $m \rho_{M}=r f$. Thus $\rho$ is a natural isomorphism.

Definition 3.13. [10] Let $M_{R}$ be a right $R$-semimodule and ${ }_{R} N$ be a left $R$-semimodule. If $F$ is the free $\mathbb{N}_{0}$-semimodule generated by the cartesian product $M \times N$ and $\sigma$ is the congruence on $F$ generated by all ordered pairs having the form $\left(\left(m+m^{\prime}, n\right),(m, n)+\left(m^{\prime}, n\right)\right),\left(\left(m, n+n^{\prime}\right),(m, n)+\left(m, n^{\prime}\right)\right)$ and $((m r, n),(m, r n))$ with $m, m^{\prime} \in M_{R}, n, n^{\prime} \in_{R} N$ and $r \in R$, then the factor semimodule $F / \sigma$ is defined to be the tensor product of $M$ and $N$ and is denoted by $M \otimes_{R} N$. When there is no confusion over the semiring, we denote the tensor product as $M \otimes N$ and the class containing $(m, n)$ by $m \otimes n$.

Proposition 3.14. Suppose $R$ is a semiring with slu $E$ and $M \in R$-Sem. Then $R \otimes M \cong M$.

Proof. Suppose $R$ is a semiring with slu $E$ and $M$ is a unitary $R$ semimodule. Consider the map $\mu: M \rightarrow R \otimes M$ defined by $m \mapsto e \otimes m$, where $m \in M$ and $e \in E$ such that $e m=m$. First we show that the definition is independent of the choice of the idempotent $e$. Suppose $e$ and $f$ are two idempotents in $R$ such that $e m=m=f m$. Let $g \in E$ be a common identity of $e$ and $f$, then $e \otimes m=g e \otimes m=g \otimes e m=g \otimes m$. Similarly $f \otimes m=g \otimes m$, hence $e \otimes m=f \otimes m$. Now it is a routine matter to verify that $\mu$ is an $R$-morphism. Also consider the map $\psi: R \otimes M \rightarrow M$ defined by $r \otimes m \mapsto r m$, where $r \in R$ and $m \in M$. Clearly $\psi$ is a well defined $R$-morphism. Now for $r \in R, m \in M$, we have

$$
(r \otimes m) \psi \mu=(r m) \mu=e \otimes r m=e r \otimes m=r \otimes m,
$$


where $e \in E$ such that $e r=r$, i.e., erm $=r m$. Also

$$
m \mu \psi=(g \otimes m) \psi=g m=m,
$$

where $g \in E$ such that $g m=m$.

Hence $\mu$ is an isomorphism, i.e., $R \otimes M \cong M$.

Suppose $R$ is a semiring with slu $E(R)$ and ${ }_{R} P$ is a unitary semimodule. Let $T$ be a subsemiring of $\operatorname{End}_{R} P$ having local units $E(T)$ such that $T \operatorname{End}_{R} P=T$ and $P \in$ Sem- $T$. Now consider the $T-R$ bisemimodule $Q=T \operatorname{Hom}_{R}(P, R) R$. Then define:

$$
\begin{aligned}
& \tau: P \otimes Q \rightarrow R \quad \text { and } \quad \mu: Q \otimes P \rightarrow T \\
& p \otimes q \mapsto p q \quad q \otimes p \mapsto q p\left(p^{\prime} \mapsto\left(p^{\prime} q\right) p\right)
\end{aligned}
$$

It is routine to verify that the maps $\tau, \mu$ are respectively $R-R$ and $T-T$ bisemimodule morphisms. Also, there is a $Q P Q$-associativity, i.e., for any $q, q^{\prime} \in Q$ and $p^{\prime} \in P, q\left(p^{\prime} q^{\prime}\right)=\left(q p^{\prime}\right) q^{\prime}$ since for any $p \in P, p\left(q\left(p^{\prime} q^{\prime}\right)\right)=$ $(p q)\left(p^{\prime} q^{\prime}\right)=\left((p q) p^{\prime}\right) q^{\prime}=\left(p\left(q p^{\prime}\right)\right) q^{\prime}=p\left(\left(q p^{\prime}\right) q^{\prime}\right)$ i.e., $q\left(p q^{\prime}\right)=(q p) q^{\prime}$.

In the notations introduced above, we obtain the following results (cf. Prop. 3.15 - 3.19) characterizing locally projective generators which are the counterparts of Prop. 3.7, 3.10, Theorem 3.11, Prop. 3.12, Corollary 3.13 respectively of [10] in our setting.

Proposition 3.15. ${ }_{R} P$ is locally projective and $P f$ is finitely generated for all $f \in E(T)$ if and only if $\mu: Q \otimes P \rightarrow T$ is a surjection. Moreover, if $\mu$ is a surjection, then it is an isomorphism.

Proof. For the necessary part, let $f \in E(T)$. Then since $P f$ is finitely generated, by Prop. 3.11, there exists a finitely generated projective subsemimodule $P^{\prime}$ of $P$ such that $P f \subseteq P^{\prime}$, i.e., $P f=P f^{2} \subseteq P^{\prime} f \subseteq P f$. Therefore $P f=P^{\prime} f$, hence it is projective (since $P^{\prime} f$ being a retract of $P^{\prime}$ is projective). Therefore by Prop. 3.7, there exists a retraction $\phi:(R e)^{n} \rightarrow P f$ for some $n \in \mathbb{N}, e^{2}=e \in R$ with coretraction $\psi: \operatorname{Pf} \rightarrow(R e)^{n}$, i.e., $\psi \phi=i d_{P f}$. Consider $e_{i} \in(R e)^{n}$ with $e$ as the $i$ th coordinate and all others being 0 for each $i=1,2, \ldots, n$, then for the canonical projections $\pi_{i}:(R e)^{n} \rightarrow R e$ we have $\sum_{i=1}^{n} x \pi_{i} e_{i}=x$ for all $x \in(R e)^{n}$. Let $p_{i}=e_{i} \phi$ and $\alpha_{i}=\pi \psi \pi_{i}$ for each $i=1,2, \ldots, n$ where $\pi:{ }_{R} P \rightarrow_{R} P f$ is given by $p \mapsto p f$. Now if we put $q_{i}=f \alpha_{i} e \in T \operatorname{Hom}_{R}(P, R) R=Q$, for all $i=1,2, \ldots, n$. Then for any $p \in P$, we have $p q_{i}=p\left(f \alpha_{i} e\right)=\left((p f) \alpha_{i}\right) e=$ 
$\left((p f)\left(\pi \psi \pi_{i}\right)\right) e=(p f)\left(\psi \pi_{i}\right)$ for all $i=1,2, \ldots, n$. Therefore for any $p \in P$,

$$
\begin{aligned}
p \sum_{i=1}^{n} q_{i} p_{i} & =\sum_{i=1}^{n} p\left(q_{i} p_{i}\right)=\sum_{i=1}^{n}\left(p q_{i}\right) p_{i}=\sum_{i=1}^{n}\left((p f)\left(\psi \pi_{i}\right)\right)\left(e_{i} \phi\right) \\
& =\left(\sum_{i=1}^{n}(p f) \psi \pi_{i} e_{i}\right) \phi=(p f) \psi \phi=p f
\end{aligned}
$$

i.e., $f=\sum_{i=1}^{n} q_{i} p_{i}$. Now for any $t \in T$ there exists an idempotent $f=\sum_{i=1}^{n} q_{i} p_{i}$ such that $t=f t$. Then we have $t=f t=\sum_{i=1}^{n} q_{i} p_{i} t=$ $\mu\left(\sum_{i=1}^{n} q_{i} \otimes p_{i} t\right)$. Thus $\mu$ is onto. Conversely, for any idempotent $f \in T$, there exist $p_{i} \in P, q_{i} \in Q$ for $i=1,2, \ldots, n$ such that $\mu\left(\sum_{i=1}^{n} q_{i} \otimes p_{i}\right)=$ $\sum_{i=1}^{n} q_{i} p_{i}=f$. Let $e \in E(R)$ such that $q_{i} e=q_{i}$ for all $i=1,2, \ldots, n$. Then we define $\alpha:(R e)^{n} \rightarrow \operatorname{Pf}$ by $\left(x_{1}, x_{2}, \ldots, x_{n}\right) \mapsto \sum_{i=1}^{n} x_{i} p_{i} f$ and $\beta: P f \rightarrow(R e)^{n}$ by $y \mapsto\left(y q_{1}, y q_{2}, \ldots, y q_{n}\right)$. Then for $y \in P f$,

$$
\begin{aligned}
y \beta \alpha & =\left(y q_{1}, y q_{2}, \ldots, y q_{n}\right) \alpha=\sum_{i=1}^{n}\left(y q_{i}\right) p_{i} f=\sum_{i=1}^{n}\left(\left(y q_{i}\right) p_{i}\right) f \\
& =\sum_{i=1}^{n} y\left(q_{i} p_{i}\right) f=y\left(\sum_{i=1}^{n} q_{i} p_{i}\right) f=y f^{2}=y,
\end{aligned}
$$

i.e., $\beta \alpha=i d_{P f}$. Hence $P f$ being a retract of $(R e)^{n}$ is finitely generated projective (by Prop. 3.7). Also, $P=\underline{\lim }_{R} P f$ (can be proved along the same lines as Prop. 3.10). Therefore ${ }_{R} P$ is locally projective.

Now let $\mu$ be a surjection and $\mu\left(\sum_{i=1}^{m} q_{i} \otimes p_{i}\right)=\mu\left(\sum_{j=1}^{n} q_{j}^{\prime} \otimes p_{j}^{\prime}\right)$. Since $P_{T}$ is unitary there exists $f \in E(T)$ such that $p_{i} f=p_{i}, p_{j}^{\prime} f=p_{j}^{\prime}$ for all $i=1,2, \ldots, m$ and $j=1,2, \ldots, n$. Now by the surjectivity of $\mu, f=\sum_{l=1}^{k} y_{l} x_{l}$, where $x_{l} \in P, y_{l} \in Q$ for all $l=1,2, \ldots, k$. Then we have

$$
\begin{aligned}
\sum_{i=1}^{m} q_{i} \otimes p_{i} & =\sum_{i=1}^{m} q_{i} \otimes p_{i}\left(\sum_{l=1}^{k} y_{l} x_{l}\right)=\sum_{i, l} q_{i} \otimes p_{i}\left(y_{l} x_{l}\right)=\sum_{i, l} q_{i} \otimes\left(p_{i} y_{l}\right) x_{l} \\
& =\sum_{i, l} q_{i}\left(p_{i} y_{l}\right) \otimes x_{l}=\sum_{i, l}\left(q_{i} p_{i}\right) y_{l} \otimes x_{l}=\sum_{l}\left(\sum_{i} q_{i} p_{i}\right) y_{l} \otimes x_{l} \\
& =\sum_{l}\left(\sum_{j} q_{j}^{\prime} p_{j}^{\prime}\right) y_{l} \otimes x_{l}=\cdots=\sum_{j=1}^{n} q_{j}^{\prime} \otimes p_{j}^{\prime},
\end{aligned}
$$

which proves that $\mu$ is injective. Hence $\mu$ is an isomorphism. 
Proposition 3.16. ${ }_{R} P$ is a generator for $R$-Sem if and only if $\tau: P \otimes Q \rightarrow$ $R$ is a surjection. Moreover, if $\tau$ is a surjection, then it is an isomorphism.

Proof. For the necessary part, since ${ }_{R} P$ is a generator, ${ }_{R} R$ is a sum of homomorphic images of $P$, i.e., every $r \in R$ can be written as $r=$ $\sum_{i=1}^{k} p_{i} \phi_{i}, p_{i} \in P, \phi_{i} \in \operatorname{Hom}_{R}(P, R)$ for all $i=1,2, \ldots, k$. Now, since $P_{T}$ is unitary, there exists $f \in E(T)$ such that $p_{i} f=p_{i}$ for all $i=1,2, \ldots, k$, also there exists $e \in E(R)$ such that $r e=r$. Therefore we have

$$
\begin{aligned}
r & =\left(\sum_{i=1}^{k} p_{i} \phi_{i}\right) e=\sum_{i=1}^{k}\left(p_{i} \phi_{i}\right) e=\sum_{i=1}^{k} p_{i}\left(\phi_{i} e\right) \\
& =\sum_{i=1}^{k}\left(p_{i} f\right)\left(\phi_{i} e\right)=\sum_{i=1}^{k} p_{i}\left(f \phi_{i} e\right)=\tau\left(\sum_{i=1}^{k} p_{i} \otimes f \phi_{i} e\right),
\end{aligned}
$$

where $f \phi_{i} e \in T \operatorname{Hom}_{R}(P, R) R=Q$. Therefore $\tau$ is onto. Conversely, let $\tau$ be a surjection then $R=\sum_{q \in Q} P q \subseteq \sum_{q \in \operatorname{Hom}_{R}(P, R)} P q=\operatorname{tr}(P)$, therefore $R=\operatorname{tr}(P)$. Hence ${ }_{R} P$ is a generator for $R$-Sem.

Now if we assume $\tau$ to be surjective, then the injectivity of $\tau$ can be proved in a manner similar to that of $\mu$ in Prop. 3.15.

Combining the above two results we obtain the following result.

Proposition 3.17. ${ }_{R} P$ is a locally projective generator and ${ }_{R} P f$ is finitely generated for all $f \in E(T)$ if and only if $\mu: Q \otimes P \rightarrow T$ and $\tau: P \otimes Q \rightarrow R$ are $T-T$ and $R-R$ isomorphisms respectively.

Proposition 3.18. Let ${ }_{R} P$ be a locally projective generator for $R$-Sem and ${ }_{R} P f$ be finitely generated for all $f \in E(T)$. Then the following hold:

(1) $R \cong\left(\operatorname{End}_{T} P\right) R \cong R \operatorname{End}_{T} Q$ as semirings.

(2) $Q:=T \operatorname{Hom}_{R}(P, R) R \cong \operatorname{Hom}_{T}(P, T) R$ as $T$-R-bisemimodules.

(3) $P \cong R \operatorname{Hom}_{T}(Q, T)$ as $R$-T-bisemimodules.

(4) $P \cong\left(\operatorname{Hom}_{R}(Q, R)\right) T$ as $R$-T-bisemimodules.

(5) $T \cong\left(\operatorname{End}_{R} Q\right) T$ as semirings.

Proof. (1) Consider the map $\sigma: R \rightarrow \operatorname{End}_{T} P$ defined by $\sigma(r)(p):=r p$, where $r \in R, p \in P$. For any $r_{1}, r_{2} \in R, p \in P, \sigma\left(r_{1}+r_{2}\right) p=\left(r_{1}+r_{2}\right) p=$ $r_{1} p+r_{2} p=\sigma\left(r_{1}\right)(p)+\sigma\left(r_{2}\right)(p)=\left(\sigma\left(r_{1}\right)+\sigma\left(r_{2}\right)\right) p$, i.e., $\sigma\left(r_{1}+r_{2}\right)=$ $\sigma\left(r_{1}\right)+\sigma\left(r_{2}\right)$. Also $\sigma\left(r_{1} r_{2}\right)(p)=\left(r_{1} r_{2}\right) p=r_{1}\left(r_{2} p\right)=\sigma\left(r_{1}\right) \sigma\left(r_{2}\right)(p)$, i.e., $\sigma\left(r_{1} r_{2}\right)=\sigma\left(r_{1}\right) \sigma\left(r_{2}\right)$. Thus $\sigma$ is a semiring morphism. Now let $\sigma\left(r_{1}\right)=\sigma\left(r_{2}\right)$ for some $r_{1}, r_{2} \in R$. Therefore $r_{1} p=r_{2} p$ for all $p \in P$. Suppose $e \in E(R)$ such that $r_{1}=r_{1} e, r_{2}=r_{2} e$. Now using Prop. 3.16, 
there exist $p_{k} \in P, q_{k} \in Q$ for $k=1,2, \ldots, n$ such that $\sum_{k=1}^{n} p_{k} q_{k}=e$. Therefore, $r_{1}=r_{1} e=r_{1} \sum_{k=1}^{n} p_{k} q_{k}=\sum_{k=1}^{n}\left(r_{1} p_{k}\right) q_{k}=\sum_{k=1}^{n}\left(r_{2} p_{k}\right) q_{k}=$ $r_{2} \sum_{k=1}^{n} p_{k} q_{k}=r_{2} e=r_{2}$. Hence $\sigma$ is injective. Therefore identifying $R$ with the subsemiring $\sigma(R)$ of $\operatorname{End}_{T} P$, let $\psi \in\left(\operatorname{End}_{T} P\right) R$, then there exists an idempotent $e^{\prime}=\sum_{i=1}^{m} p_{i}^{\prime} q_{i}^{\prime} \in R$, such that $\psi e^{\prime}=\psi$. Then for any $p \in P$ we have

$$
\begin{aligned}
\psi(p) & =\left(\psi e^{\prime}\right) p=\psi\left(e^{\prime} p\right)=\psi\left(\sum_{i=1}^{m}\left(p_{i}^{\prime} q_{i}^{\prime}\right) p\right)=\psi\left(\sum_{i=1}^{m} p_{i}^{\prime}\left(q_{i}^{\prime} p\right)\right) \\
& =\sum_{i=1}^{m} \psi\left(p_{i}^{\prime}\right)\left(q_{i}^{\prime} p\right)=\sum_{i=1}^{m}\left(\psi\left(p_{i}^{\prime}\right) q_{i}^{\prime}\right) p=\sigma\left(\sum_{i=1}^{m}\left(\psi\left(p_{i}^{\prime}\right) q_{i}^{\prime}\right)\right)(p),
\end{aligned}
$$

i.e., $\psi=\sigma\left(\sum_{i=1}^{m}\left(\psi\left(p_{i}^{\prime}\right) q_{i}^{\prime}\right)\right)$. Thus $R \cong\left(\operatorname{End}_{T} P\right) R$ as semirings. Similarly, considering the map $\xi: R \rightarrow \operatorname{End}_{T} Q$ defined by $\xi(r)(q):=q r$ we can show that $R \cong R \operatorname{End}_{T} Q$ as semirings.

(2) Define the map $\lambda: Q \rightarrow \operatorname{Hom}_{T}(P, T) R$ by $\lambda(q)(p):=q p$, where $q \in Q, p \in P$. For $q \in Q$ there exists $e^{\prime} \in E(R)$ such that $q e^{\prime}=q$, therefore using the $Q R P$-associativity $\left(\lambda(q) e^{\prime}\right) p=\lambda(q)\left(e^{\prime} p\right)=q\left(e^{\prime} p\right)=$ $\left(q e^{\prime}\right) p=q p=\lambda(q)(p)$, i.e., $\lambda(q)=\lambda(q) e^{\prime} \in \operatorname{Hom}_{T}(P, T) R$. That $\lambda$ is a monoid morphism follows from the fact that $\mu$ is a monoid morphism and using the $Q R P$-associativity we have $(t \lambda(q) r)(p)=t \lambda(q)(r p)=t(q(r p))=$ $t((q r) p)=(t q r) p=\lambda(t q r)(p)$. Thus $\lambda$ is a $T$ - $R$ morphism. For $q, q^{\prime} \in Q$, let $\lambda(q)=\lambda\left(q^{\prime}\right)$ then for any $p \in P, q p=q^{\prime} p$. Suppose $e^{2}=e \in R$ such that $q=q e, q^{\prime}=q^{\prime} e$. Now, in view of Prop. 3.16, there exist $p_{k} \in P, q_{k} \in Q$ for $k=1,2, \ldots, n$ such that $\sum_{k=1}^{n} p_{k} q_{k}=e$. Therefore, $q=q e=q \sum_{k=1}^{n} p_{k} q_{k}=\sum_{k=1}^{n}\left(q p_{k}\right) q_{k}=\sum_{k=1}^{n}\left(q^{\prime} p_{k}\right) q_{k}=q^{\prime} \sum_{k=1}^{n} p_{k} q_{k}=$ $q^{\prime} e=q^{\prime}$. Let $\phi \in \operatorname{Hom}_{T}(P, T) R$, then there exists $e^{\prime}=\sum_{i=1}^{m} p_{i}^{\prime} q_{i}^{\prime} \in E(R)$, such that $\phi e^{\prime}=\phi$. Then using $T Q P$-associativity, for any $p \in P$ we have

$$
\begin{aligned}
\phi(p) & =\left(\phi e^{\prime}\right) p=\phi\left(e^{\prime} p\right)=\phi\left(\sum_{i=1}^{m}\left(p_{i}^{\prime} q_{i}^{\prime}\right) p\right)=\phi\left(\sum_{i=1}^{m} p_{i}^{\prime}\left(q_{i}^{\prime} p\right)\right) \\
& =\sum_{i=1}^{m} \phi\left(p_{i}^{\prime}\right)\left(q_{i}^{\prime} p\right)=\sum_{i=1}^{m}\left(\phi\left(p_{i}^{\prime}\right) q_{i}^{\prime}\right) p=\lambda\left(\sum_{i=1}^{m}\left(\phi\left(p_{i}^{\prime}\right) q_{i}^{\prime}\right)\right)(p),
\end{aligned}
$$

i.e., $\phi=\lambda\left(\sum_{i=1}^{m}\left(\phi\left(p_{i}^{\prime}\right) q_{i}^{\prime}\right)\right)$. Thus $\lambda$ is an isomorphism.

$(3),(4)$ can be proved in a manner similar to (2) and (5) can be proved along the same lines as (1).

Proposition 3.19. Let ${ }_{R} P$ be a locally projective generator for $R$-Sem and ${ }_{R} P f$ be finitely generated for all $f \in E(T)$. Then ${ }_{T} Q \in T$-Sem, $P_{T} \in$ 
Sem- $T, Q_{R} \in$ Sem- $R$ are locally projective generators for their respective categories.

Proof. Suppose that ${ }_{R} P \in R$-Sem is a locally projective generator for $R$-Sem and ${ }_{R} P f$ is finitely generated for all $f^{2}=f \in T$. Then by Prop. 3.18, identifying $P$ with $R \operatorname{Hom}_{T}(Q, T)$ and $R$ with $R \operatorname{End}_{T} Q$ and using the fact that $\tau$ and $\mu$ are isomorphisms (Prop. 3.17) and finally applying Prop. 3.17 to ${ }_{T} Q$, we have that ${ }_{T} Q \in T$-Sem is a locally projective generator. Similarly $P_{T}, Q_{R}$ can be proved to be locally projective generators for their respective categories.

\section{Morita equivalence and Morita context}

Definition 4.1. Let $R, S$ be two semirings with local units. We call $R$ and $S$ to be Morita equivalent if the categories $R$-Sem and $S$-Sem are equivalent, i.e., there exist additive functors $F: R$-Sem $\rightarrow S$-Sem and $G: S$-Sem $\rightarrow R$-Sem such that $F$ and $G$ are mutually inverse equivalence functors.

In what follows by equivalence functors we mean additive equivalence functors. In this section we are going to characterize Morita equivalence for semirings with local units (cf. Theorem 4.13). In order to achieve this we first obtain some results below.

Definition 4.2. A unitary bisemimodule ${ }_{R} P_{S}$ is said to be faithfully balanced if the canonical homomorphisms $S \rightarrow \operatorname{End}_{R} P$ and $R \rightarrow \operatorname{End}_{S} P$ given by $s \mapsto \rho_{s}(p \mapsto p s)$ and $r \mapsto \lambda_{r}(p \mapsto r p)$ respectively, where $s \in S, r \in R, p \in P$, are injective and identifying $R$ and $S$ with the corresponding subsemirings of endomorphisms of $P, S \operatorname{End}_{R} P=S$ and $\left(\operatorname{End}_{S} P\right) R=R$.

The following result is analogous to the case of categories of semimodules over a semiring with identity [10] and can be proved in a similar manner.

Lemma 4.3. Let $F: R$-Sem $\rightleftarrows S$-Sem $: G$ be an equivalence of the categories $R$-Sem and $S$-Sem, and $\theta$ be a surjection in $R$-Sem. Then $F(\theta)$ is a surjection in $S$-Sem.

Lemma 4.4. Let $F: R$-Sem $\rightleftarrows S$-Sem $: G$ be an equivalence of the categories $R$-Sem and $S$-Sem, and ${ }_{R} P \in R$-Sem be projective. Then $F(P) \in S$-Sem is projective, too. 
Proof. By Lemma 4.3, $F$ preserves surjections. So in view of the definition of projectivity in the category of unitary semimodules [cf. Def. 3.1], with relevant modification of the proof of [9, Proposition 5.1.34] by replacing the notion of epimorphism by surjectivity, the result follows easily.

Lemma 4.5. Let $F: R$-Sem $\rightleftarrows S$-Sem $: G$ be an equivalence of the categories $R$-Sem and $S$-Sem, and ${ }_{R} P \in R$-Sem be a generator for $R$-Sem. Then $F(P) \in S$-Sem is a generator for $S$-Sem.

Proof. Let $N \in S$-Sem. Since $P$ is a generator, there exists a surjection $\alpha: P^{(I)} \rightarrow G(N)$ for some non-empty index set $I$. By Lemma 4.3, $F(\alpha)$ : $F\left(P^{(I)}\right) \rightarrow F G(N)$ is a surjection where $F G(N) \cong N$. Also $F$ and $G$ being mutually inverse equivalence functors, by [9, Prop. 5.1.31], $G$ is the right adjoint of $F$. Then by the dual of [12, Theorem 5.5.1], $F$ preseves direct limits, hence preserves coproducts, i.e., $F\left(P^{(I)}\right) \cong F(P)^{(I)}$. Thus $N$ is a homomorphic image of a direct sum of copies of $F(P)$. Hence $F(P)$ is a generator for $S$-Sem.

We skip the proof of Lemma 4.6 and Lemma 4.7 as they can be proved along the same lines as in the case of module theory [3].

Lemma 4.6. Let $F: R$-Sem $\rightarrow S$-Sem be a categorical equivalence. Then for each $M, M^{\prime} \in R$-Sem the restriction of $F$ to $\operatorname{Hom}_{R}\left(M, M^{\prime}\right)$, $F: \operatorname{Hom}_{R}\left(M, M^{\prime}\right) \rightarrow \operatorname{Hom}_{S}\left(F(M), F\left(M^{\prime}\right)\right)$ is a monoid isomorphism. In particular $F: \operatorname{End}_{R}(M) \rightarrow \operatorname{End}_{S}(F(M))$ is a semiring isomorphism.

Lemma 4.7. Let $F: R$-Sem $\rightarrow S$-Sem be an equivalence of the categories $R$-Sem and $S$-Sem, and let ${ }_{R} P \in R$-Sem be finitely generated. Then $F(P) \in S$-Sem is finitely generated, too.

Theorem 4.8. Let $F: R$-Sem $\rightleftarrows S$-Sem : $G$ be an equivalence of the categories $R$-Sem and $S$-Sem, and let ${ }_{R} P \in R$-Sem be a locally projective generator. Then $F(P) \in S$-Sem is a locally projective generator, too.

Proof. By [9, Prop. 5.1.31], $G$ is the right adjoint of $F$. Then by the dual of [12, Theorem 5.5.1], $F$ preseves direct limits. Using this fact together with Lemmas 4.4, 4.5 and 4.7 we obtain the result.

In the following proposition we observe the adjointness of the tensor functor and Hom functor between the categories of unitary semimodules. It is a routine verification so we omit the proof. 
Proposition 4.9. Let $R, S$ be semirings with local units and ${ }_{S} A_{R} \in$ $S$-Sem- $R,{ }_{R} B \in R$-Sem, ${ }_{S} C \in S$-Sem. Then

$$
\phi: \operatorname{Hom}_{S}(A \otimes B, C) \rightarrow \operatorname{Hom}_{R}\left(B, R \operatorname{Hom}_{S}(A, C)\right)
$$

given by

$$
\begin{aligned}
\alpha \mapsto \alpha^{\prime}: B & \rightarrow R \operatorname{Hom}_{S}(A, C) \\
b & \mapsto b \alpha^{\prime}: A \rightarrow C \\
& a \mapsto(a \otimes b) \alpha
\end{aligned}
$$

is a bijective mapping natural in ${ }_{S} A_{R},{ }_{R} B,{ }_{S} C$. In particular, the functor $R \operatorname{Hom}_{S}(A,-)$ is right adjoint to the functor $A \otimes-$.

The next result is the counterpart of Theorem 4.5 of [10] in this general setting.

Theorem 4.10. For a functor $F: R$-Sem $\rightarrow S$-Sem the following statements are equivalent.

(1) F has a right adjoint.

(2) F preserves direct limits.

(3) There exists a unitary $S$-R-bisemimodule $Q$ such that the functors $Q \otimes-: R$-Sem $\rightarrow S$-Sem and $F$ are naturally isomorphic.

Proof. (1) $\Rightarrow(2)$ and (3) $\Rightarrow$ (1) follow from the right analogue of [12, Theorem 5.5.1] and Prop. 4.9 respectively.

(2) $\Rightarrow(3)$ Let $Q:=F(R) \in S$-Sem. Then $F$ induces a right $R$ semimodule structure on $Q$ with the $R$-action given by $Q \times R \rightarrow Q$ by $(q, r) \mapsto q F\left(\rho_{r}\right)$ where $\rho_{r}: R \rightarrow R$ is given by $x \mapsto x r$. In order to show that $Q_{R}$ is unitary, suppose $q \in Q$. Now $Q=F(\underset{e \in E(R)}{\cup} R e)=\underset{e \in E(R)}{\cup} F(R e)$ (since $R$ is a semiring with local units, union coincides in this formula with direct limit and by the hypothesis $F$ preserves direct limits). Therefore $q \in F(R e)$ for some idempotent $e \in R$. Then we have $q e=q F\left(\rho_{e}\right)=q$ (since $\rho_{e}=1_{R e}$ implies that $\left.F\left(\rho_{e}\right)=1_{F(R e)}\right)$. Thus $Q$ is a unitary $S$ $R$-bisemimodule. Then the proof follows similarly as in [10, Theorem 4.5].

Theorem 4.11. Let $R$ and $S$ be Morita equivalent semirings with local units via inverse equivalences $F: R$-Sem $\rightarrow S$-Sem and $G: S$-Sem $\rightarrow$ $R$-Sem. Set $P=G(S)$ and $Q=F(R)$. Then the following hold:

(1) ${ }_{R} P_{S},{ }_{S} Q_{R}$ are unitary faithfully balanced bisemimodules.

(2) ${ }_{R} P, P_{S},{ }_{S} Q, Q_{R}$ are locally projective generators.

(3) $F \cong Q \otimes-, G \cong P \otimes-$. 
(4) $F \cong S \operatorname{Hom}_{R}(P,-), G \cong R \operatorname{Hom}_{S}(Q,-)$.

(5) ${ }_{R} P_{S} \cong R \operatorname{Hom}_{S}(Q, S) \cong\left(\operatorname{Hom}_{R}(Q, R)\right) S$ and

$$
{ }_{S} Q_{R} \cong S \operatorname{Hom}_{R}(P, R) \cong \operatorname{Hom}_{S}(P, S) R
$$

Proof. Let $G(S)=P$, then $G$ being an equivalence functor using Lemma 4.6 we have $\operatorname{End}_{S} S \cong \operatorname{End}_{R} P$ as semirings. By Prop. 3.12, $S \cong S \operatorname{End}_{S} S$ as semirings. Since $P$ is a right $\operatorname{End}_{R} P$-semimodule, identifying $S$ with the subsemiring $S \operatorname{End}_{S} S$ of $\operatorname{End}_{S} S, P$ can be considered as a right $S$ semimodule with the action $P \times S \rightarrow P$ given by $(p, s) \mapsto p G\left(\rho_{s}\right)$ where $\rho_{s}: S \rightarrow S$ is given by $t \mapsto t s$. That $P_{S}$ is unitary follows similarly as in the proof of Theorem 4.10. Thus $P$ is a unitary $R$ - $S$-bisemimodule. Now since $S$ is a locally projective generator, by Theorem $4.8,{ }_{R} P=G(S)$ is a locally projective generator. In view of Lemma 4.7, $P f=G(S f)$ is a finitely generated left $S$-semimodule for all $f \in E(S)$ and $S \cong S \operatorname{End}_{S} S \cong$ $S \operatorname{End}_{R} P$ as semirings. Since ${ }_{R} P$ is a locally projective generator with $P f$ finitely generated for all $f^{2}=f \in S$, using (1) of Prop. 3.18 we have $R \cong\left(\operatorname{End}_{S} P\right) R$ as semirings. Hence ${ }_{R} P_{S}$ is a faithfully balanced bisemimodule. Similarly $Q=F(R)$ is a unitary faithfully balanced $S$ - $R$ bisemimodule. Hence (1) is proved.

Since $F$ and $G$ are mutually inverse equivalence functors, they are adjoint to each other [9, Prop. 5.1.31]. Therefore using Theorem 4.10, we obtain $F \cong Q \otimes-$. Similarly $G \cong P \otimes-$. By Prop. 4.9, $Q \otimes-$ is left adjoint to $R \operatorname{Hom}_{S}(Q,-)$ and $P \otimes-$ is left adjoint to $S \operatorname{Hom}_{R}(P,-)$. Then by uniqueness of adjoint functors upto natural isomorphism [9, Cor. 5.1.10], we obtain $F \cong Q \otimes-\cong S \operatorname{Hom}_{R}(P,-)$ and $G \cong P \otimes-\cong R \operatorname{Hom}_{S}(Q,-)$. This proves (3) and (4).

Now using (4) we obtain, $P=G(S) \cong R \operatorname{Hom}_{S}(Q, S)$ as $R$-Sbisemimodule and $Q=F(R) \cong S \operatorname{Hom}_{R}(P, R)$ as $S$-R-bisemimodule. Since by (1), $Q_{R}$ is unitary, using Prop. 3.18 we obtain, $Q=$ $Q R \cong S \operatorname{Hom}_{R}(P, R) R \cong \operatorname{Hom}_{S}(P, S) R$ as $S$-R-bisemimodule and also $P \cong\left(\operatorname{Hom}_{R}(Q, R)\right) S$ as $R$-S-bisemimodule, which proves (5). Now (2) clearly follows from Prop. 3.19.

Definition 4.12. [15] Let $R$ and $S$ be two semirings and ${ }_{R} P_{S}$ and ${ }_{S} Q_{R}$ be an $R$-S-bisemimodule and an $S$-R-bisemimodule, respectively and $\tau$ : $P \otimes_{S} Q \rightarrow R$ and $\mu: Q \otimes_{R} P \rightarrow S$ be an $R$-S-bisemimodule homomorphism and an $S$-R-bisemimodule homomorphism, respectively, such that $\tau(p \otimes$ $q) p^{\prime}=p \mu\left(q \otimes p^{\prime}\right)$ and $\mu(q \otimes p) q^{\prime}=q \tau\left(p \otimes q^{\prime}\right)$ for all $p, p^{\prime} \in P$ and $q, q^{\prime} \in Q$. Then the sixtuple $(R, S, U, V, \tau, \mu)$ is called a Morita context for semirings. 
Moreover, we say that a Morita context is unitary if ${ }_{R} P_{S}$ and ${ }_{S} Q_{R}$ are unitary bisemimodules.

Theorem 4.13. Let $R$ and $S$ be semirings with local units. Then the following are equivalent:

(1) $R$ and $S$ are Morita equivalent.

(2) There exists a faithfully balanced unitary bisemimodule ${ }_{R} P_{S}$ such that ${ }_{R} P$ is a locally projective generator and ${ }_{R} P f$ is finitely generated for all $f \in E(S)$.

(3) There exists a unitary Morita context $\left(R, S,{ }_{R} P_{S},{ }_{S} Q_{R}, \tau, \mu\right)$ with surjective $\tau, \mu$.

(4) There exists a unitary Morita context $\left(R, S,{ }_{R} P_{S}, S Q_{R}, \tau, \mu\right)$ with bijective $\tau, \mu$.

Proof. (1) $\Rightarrow(2)$ Let $P:=G(S)$. Then the proof follows from Theorem 4.11 .

$(2) \Rightarrow(3)$ Suppose there exists a unitary bisemimodule ${ }_{R} P_{S}$ such that ${ }_{R} P$ is a locally projective generator and ${ }_{R} P f$ is finitely generated for all $f \in E(S)$ and $S \cong S \operatorname{End}_{R} P$ as semirings. Let $Q=S \operatorname{Hom}_{R}(P, R) R$. Then define:

$$
\begin{aligned}
& \tau: P \otimes Q \rightarrow R \quad \text { and } \quad \mu: Q \otimes P \rightarrow S \\
& p \otimes q \mapsto p q \quad q \otimes p \mapsto q p\left(p^{\prime} \mapsto\left(p^{\prime} q\right) p\right)
\end{aligned}
$$

It is routine to verify that the maps $\tau, \mu$ are respectively $R-R$ and $S-S$ morphisms. For any $p^{\prime} \in P$,

$$
\begin{aligned}
p^{\prime} \mu(q \otimes p) q^{\prime} & =p^{\prime}\left((q p) q^{\prime}\right)=\left(p^{\prime}(q p)\right) q^{\prime}=\left(\left(p^{\prime} q\right) p\right) q^{\prime} \\
& =\left(p^{\prime} q\right)\left(p q^{\prime}\right)=p^{\prime}\left(q\left(p q^{\prime}\right)\right)=p^{\prime}\left(q \tau\left(p \otimes q^{\prime}\right)\right),
\end{aligned}
$$

i.e., $\mu(q \otimes p) q^{\prime}=q \tau\left(p \otimes q^{\prime}\right)$. Also $\tau(p \otimes q) p^{\prime}=(p q) p^{\prime}=p\left(q p^{\prime}\right)=p \mu\left(q \otimes p^{\prime}\right)$.

Consequently $\left(R, S,_{R} P_{S}, S Q_{R}, \tau, \mu\right)$ is a Morita context. By hypothesis, ${ }_{R} P$ is a locally projective generator and ${ }_{R} P f$ is finitely generated for all $f \in E(S)$ and $S \cong S \operatorname{End}_{R} P$ as semirings. Hence using Prop. 3.16 and Prop. 3.15, we get that $\tau, \mu$ are surjections.

$(3) \Rightarrow(4)$ Suppose $\left(R, S,_{R} P_{S}, S Q_{R}, \tau, \mu\right)$ is a unitary Morita context with surjective $\tau, \mu$. Let $\tau\left(\sum_{i=1}^{m} p_{i} \otimes q_{i}\right)=\tau\left(\sum_{j=1}^{n} p_{j}^{\prime} \otimes q_{j}^{\prime}\right)$, where $p_{i}, p_{j}^{\prime} \in$ $P, q_{i}, q_{j}^{\prime} \in Q$ for all $i=1,2, \ldots, m, j=1,2, \ldots, n$. Since $Q_{R}$ is unitary, there exists an idempotent $e \in R$ such that $q_{i} e=q_{i}, q_{j}^{\prime} e=q_{j}^{\prime}$ for all $i=1,2, \ldots, m, j=1,2, \ldots, n$. Now by the surjectivity of $\tau, e=$ 
$\tau\left(\sum_{l=1}^{k} x_{l} \otimes y_{l}\right)$, where $x_{l} \in P, y_{l} \in Q$ for all $l=1,2, \ldots, k$. Therefore we have,

$$
\begin{aligned}
\sum_{i=1}^{m} p_{i} \otimes q_{i} & =\sum_{i=1}^{m} p_{i} \otimes q_{i} \tau\left(\sum_{l=1}^{k} x_{l} \otimes y_{l}\right)=\sum_{i, l} p_{i} \otimes q_{i} \tau\left(x_{l} \otimes y_{l}\right) \\
& =\sum_{i, l} p_{i} \otimes \mu\left(q_{i} \otimes x_{l}\right) y_{l}=\sum_{i, l} p_{i} \mu\left(q_{i} \otimes x_{l}\right) \otimes y_{l} \\
& =\sum_{l} \sum_{i} \tau\left(p_{i} \otimes q_{i}\right) x_{l} \otimes y_{l}=\sum_{l=1}^{k} \tau\left(\sum_{i=1}^{m} p_{i} \otimes q_{i}\right) x_{l} \otimes y_{l} \\
& =\sum_{l=1}^{k} \tau\left(\sum_{j=1}^{n} p_{j}^{\prime} \otimes q_{j}^{\prime}\right) x_{l} \otimes y_{l}=\cdots=\sum_{j=1}^{n} p_{j}^{\prime} \otimes q_{j}^{\prime}
\end{aligned}
$$

which proves that $\tau$ is injective. Similarly $\mu$ is also injective.

$(4) \Rightarrow(1)$ Let $\left(R, S,{ }_{R} P_{S}, S Q_{R}, \tau, \mu\right)$ be a unitary Morita context with bijective $\tau, \mu$. Then $P \otimes_{S} Q \cong R$ and $Q \otimes_{R} P \cong S$. Therefore for every $M \in R$-Sem, $P \otimes_{S}\left(Q \otimes_{R} M\right) \cong\left(P \otimes_{S} Q\right) \otimes_{R} M \cong R \otimes_{R} M \cong M$ (cf. Prop. 3.14). Now we consider the class of isomorphisms $\eta=\left\{\eta_{X}\right.$ : $P \otimes_{S}\left(Q \otimes_{R} X\right) \rightarrow_{R} X \mid X \in R$-Sem $\}$. Then $\eta$ is a natural isomorphism between the identity functor $1_{R \text {-Sem }}$ on the category $R$-Sem and the functor $P \otimes_{S}\left(Q \otimes_{R}-\right)$ as for all ${ }_{R} X,_{R} Y \in R$-Sem and $f \in \operatorname{Hom}_{R}(X, Y)$ the following diagram commutes:

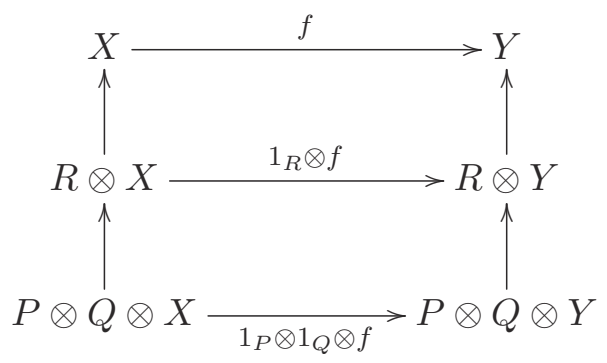

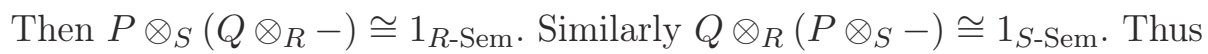
$P \otimes_{S}-: S$-Sem $\rightarrow R$-Sem $: Q \otimes_{R}-$ is an equivalence of the categories $R$-Sem and $S$-Sem.

Analogously to Corollary 4.3 of [1], we have the following proposition.

Proposition 4.14. Let $R$ be a semiring with slu. Then the following are equivalent: 
(1) $R$ is Morita equivalent to a semiring with identity.

(2) There exists an idempotent $e \in R$ such that $R=R e R$.

Proof. (1) $\Rightarrow(2)$ Suppose $R$ is Morita equivalent to a semiring $S$ with identity via inverse equivalences $F: R$-Sem $\rightleftarrows S$-Sem $: G$. Let $P=G(S)$. Since $S$ is a finitely generated projective generator, ${ }_{R} P$ also is a finitely generated projective generator. Now ${ }_{R} P$ being a finitely generated projective unitary $R$-semimodule, by Prop. 3.7, there exists a surjective $R$-morphism $\phi:(R e)^{m} \rightarrow P$ for some idempotent $e \in R$ and $m \in \mathbb{N}$ which implies that $R e$ is a generator for $R$-Sem. Also since for any $r \in R, R r$ is finitely generated, using Prop. 3.4 there exists a surjective $R$-morphism $\psi:(R e)^{n} \rightarrow R r$ for some $n \in \mathbb{N}$. Therefore there exists $\left(r_{1}, r_{2}, \ldots, r_{n}\right) \in(R e)^{n}$ such that $r=\left(r_{1}, r_{2}, \ldots, r_{n}\right) \psi=r_{1} e((e, 0, \ldots, 0) \psi)+\cdots+r_{n} e((0, \ldots, 0, e) \psi) \in$ $R e R r \subseteq R e R$, which is true for any $r \in R$. Therefore $R=R e R$.

$(2) \Rightarrow(1)$ Let $P=R e$. Then clearly $P$ is a finitely generated projective unitary $R$-semimodule. Also for any $M \in R$-Sem, for each $m \in M$ consider the map $\rho_{m}: P \rightarrow M$ defined by $y \mapsto y m$, where $y \in P, m \in M$. Then $\rho=\bigoplus_{m \in M} \rho_{m}: \bigoplus_{M} P \rightarrow M$, where $\left(\bigoplus_{M} P\right) \rho=\sum_{m \in M} P \rho_{m}=P M=$ $P(R M)=(P R) M=(\operatorname{Re} R) M=R M=M$, which implies that $\rho$ is a surjection. Thus $P$ is a finitely generated projective generator hence a locally projective generator for $R$-Sem. Now if we take $S=\operatorname{End}_{R} P=$ $\operatorname{End}_{R}(R e)=e R e$, then using (2) of Theorem 4.13, $R$ and $S=e R e$ are Morita equivalent semirings.

\section{Morita invariant properties}

In this section we discuss some properties of semirings with local units which remain invariant under Morita equivalence. The results obtained here are nothing but counterparts of the results of [17] in the setting of semirings with local units.

Theorem 5.1. Let $R$ and $S$ be Morita equivalent semirings with local units via the Morita context $\left(R, S,{ }_{R} P_{S},{ }_{S} Q_{R}, \tau, \mu\right)$. Then $R$ is additively cancellative (additively idempotent, additively regular, zero-sum free) if and only if $P$ is additively cancellative (respectively additively idempotent, additively regular, zero-sum free).

Proof. Let $R$ be additively cancellative and $a, b, c \in P$ such that $a+c=$ $b+c$. Also let $t \in S$ be an idempotent such that $a=a t, b=b t$. Then the result can be proved in a similar manner to that of [17, Theorem 2.1] by replacing $t$ in place of $1_{S}$. Other parts follow similarly from their corresponding definitions. 
Theorem 5.2. Let $R$ and $S$ be Morita equivalent semirings with local units via the Morita context $\left(R, S, R P_{S}, S Q_{R}, \tau, \mu\right)$. Then the lattice $\operatorname{Id}(R)$ of ideals of $R$ and the lattice $\operatorname{Sub}(P)$ of subsemimodules of $P$ are isomorphic. Moreover, the isomorphism takes finitely generated ideals to finitely generated subsemimodules and vice-versa.

Proof. Let us define

$$
f: \operatorname{Id}(R) \rightarrow \operatorname{Sub}(P) \quad \text { and } \quad g: \operatorname{Sub}(P) \rightarrow \operatorname{Id}(R)
$$

by

$$
f(I):=\left\{\sum_{k=1}^{n} i_{k} p_{k} \mid p_{k} \in P, i_{k} \in I \text { for all } k ; n \in \mathbb{N}\right\}
$$

and

$$
g(N):=\left\{\sum_{k=1}^{n} \tau\left(p_{k} \otimes q_{k}\right) \mid p_{k} \in N, q_{k} \in Q \text { for all } k ; n \in \mathbb{N}\right\},
$$

respectively. Then with relevant modification of the proof of Theorem 2.2 [17] by replacing the identity by a local unit the rest of the proof can be completed.

Remark 5.3. The above result has its counterpart for $k$-ideals and $h$ ideals which is analogous to Theorem 2.5 of [17].

Remark 5.4. $f$ and $g$ also preserve $k$-closure and $h$-closure.

The following result is an obvious corollary of Theorem 5.2 and the result mentioned in Remark 5.3.

Corollary 5.5. Let $R$ and $S$ be Morita equivalent semirings with local units via the Morita context $\left(R, S,{ }_{R} P_{S},{ }_{S} Q_{R}, \tau, \mu\right)$. Then $R$ is ideal-simple ( $k$-ideal simple, $h$-ideal simple) if and only if $P$ is subsemimodule-simple (respectively $k$-subsemimodule simple, $h$-subsemimodule simple).

The following result is the counterpart of Theorem 2.8 of [17] in the present setting.

Theorem 5.6. Let $R$ and $S$ be Morita equivalent semirings with local units via the Morita context $\left(R, S,{ }_{R} P_{S},{ }_{S} Q_{R}, \tau, \mu\right)$. Then $R$ is Noetherian if and only if $P$ is Noetherian. 
Theorem 5.7. Let $R$ and $S$ be Morita equivalent semirings with local units via the Morita context $\left(R, S, R P_{S}, S Q_{R}, \tau, \mu\right)$. Then the lattices $\operatorname{Con}(R)$ and $\operatorname{Con}(P)$ of congruences of $R$ and $P$ respectively are isomorphic. Moreover the isomorphism takes Bourne congruences to Bourne congruences, Iizuka congruences to Iizuka congruences and ring congruences to module congruences and vice-versa.

Proof. Let us define

$$
\alpha: \operatorname{Con}(R) \rightarrow \operatorname{Con}(P) \quad \text { by } \quad \alpha(\rho):=\alpha_{\rho}^{\operatorname{tr}}
$$

and

$$
\beta: \operatorname{Con}(P) \rightarrow \operatorname{Con}(R) \quad \text { by } \quad \beta(\sigma):=\beta_{\sigma}{ }^{\text {tr }},
$$

where

$$
\alpha_{\rho}=\left\{\left(\sum_{k=1}^{n} r_{k} p_{k}, \sum_{k=1}^{n} r_{k}^{\prime} p_{k}\right) \mid\left(r_{k}, r_{k}^{\prime}\right) \in \rho, p_{k} \in P \text { for all } k ; n \in \mathbb{N}\right\}
$$

and

$\beta_{\sigma}=\left\{\left(\sum_{k=1}^{n} \tau\left(p_{k} \otimes q_{k}\right), \sum_{k=1}^{n} \tau\left(p_{k}^{\prime} \otimes q_{k}\right)\right) \mid\left(p_{k}, p_{k}^{\prime}\right) \in \sigma, q_{k} \in Q\right.$ for all $\left.k ; n \in \mathbb{N}\right\}$.

The rest of the proof is a slight modification of the proof of Theorem 2.10 of [17].

The following result is an obvious corollary of the above theorem.

Corollary 5.8. Let $R$ and $S$ be Morita equivalent semirings with local units via the Morita context $\left(R, S,{ }_{R} P_{S}, Q_{R}, \tau, \mu\right)$. Then $R$ is (Bourne, Iizuka, ring) congruence-simple if and only if $P$ is (Bourne, Iizuka, module) congruence-simple.

\section{Concluding remark}

All the above results in section 5 investigate relationship between $R$ and $P$. But similar relationship can be established between $R$ and $Q, S$ and $P, S$ and $Q$ i.e., Theorems 5.1, 5.2, 5.6, 5.7 have their counterparts for other pairs of the components of Morita equivalent semirings with local units. Since a semiring with identity is also a semiring with local units, Theorems 4.11, 4.13 include some of the results of Theorems 4.6, 4.8 of [15]. 


\section{Acknowledgement}

The authors are very much thankful to the learned referee for his meticulous referring and subsequent detection of some errors both in mathematics as well as presentation whose compliance has made the paper what it is now. The authors are also grateful to Prof. L. Márki and Prof. P. N. Ánh of Alfréd Rényi Institute of Mathematics, Hungary for a necessary discussion in order to resolve one issue raised by the learned referee. Lastly the authors would like to acknowledge the encouragement provided by Prof. Y. Katsov of Hanover College, USA at the time of preparation of the paper.

\section{References}

[1] G. D. Abrams, Morita equivalence for rings with local units, Communications in Algebra, 11(8), 1983, 801-837.

[2] J. Adamek, H. Herlich and G. Strecker, Abstract and concrete categories, John Wiley \& Sons, Inc., New York, 1990.

[3] F. W. Anderson and K. R. Fuller, Rings and categories of modules, 2nd ed., Springer, New York, 1992.

[4] P. N. Ánh and L. Márki, Morita equivalence for rings without identity, Tsukuba J. Math., 11(1), 1987, 1-16.

[5] S. Ghosh, A characterization of ring congruences on semirings, Soochow J. Math., 19(3), 1993, 305-312.

[6] J. S. Golan, Semirings and Their Applications, Kluwer Academic Publishers, Dordrecht, 1999.

[7] U. Hebisch and H. J. Weinert, Semirings : Algebraic Theory and Applications in Computer Science, World Scientific Publishing, Singapore, 1998.

[8] U. Hebisch and H. J. Weinert, Semirings and semifields, Handbook of Algebra, Vol. 1, 1996, 425-462.

[9] M. Kilp, U. Knauer and A. V. Mikhalev, Monoids, acts and categories, Walter de Gruyter, Berlin, New York, 2000.

[10] Y. Katsov and T. G. Nam, Morita equivalence and homological characterization of semirings, Journal of Algebra and Its Applications, 10 (3), 2011, 445-473.

[11] Y. Katsov, T. G. Nam and J. Zumbragel, On simpleness of semirings and complete semirings, J. Algebra Appl., 13(6), 2014, 1450015 29pp.

[12] S. Mac Lane, Categories for the Working Mathematician, Springer, New York, 1971.

[13] K. Morita, Duality of modules and its applications to the theory of rings with minimum condition, Science Rep. Tokyo Kyoiku Daigaku Sect., A6, 1958, 85-142.

[14] T. K. Mukherjee, M. K. Sen and S. Ghosh, Chain conditions on semirings, International Journal of Mathematics and Mathematical Sciences, 19(2), 1996, 321-326. 
[15] S. K. Sardar, S. Gupta and B.C. Saha, Morita equivalence of semirings and its connection with Nobusawa $\Gamma$-semirings with unities, Algebra Colloquium, 22 (Spec 1), 2015, 985-1000.

[16] S. K. Sardar and S. Gupta, Morita invariants of semirings, J. Agebra Appl., 15(2), 2016, 14pp : 1650023.

[17] S. K. Sardar and S. Gupta, Morita invariants of semirings-II, Asian-European Journal of Mathematics, 11(1), 2018: 1850014.

\section{CONTACT INFORMATION}

Monali Das, Department of Mathematics,

Sujit Kumar Jadavpur University, Kolkata, India

Sardar

E-Mail(s): monali.ju7@gmail.com,

sksardarjumath@gmail.com,

sujitk.sardar@jadavpuruniversity.in

Sugato Gupta Department of Mathematics,

Vidyasagar College for Women, Kolkata,

India

E-Mail(s): sguptaju@gmail.com

Received by the editors: 16.11.2018

and in final form 02.09.2019. 